\title{
Theory of equidistance and betweenness relations in regular metric spaces *
}

\section{Moszyńska (Warszawa)}

Abstract. The paper contains some partial results concerning theory of metric equidistance and betweenness relations.

The purpose of this note is, roughly speaking, to describe metric spaces in terms of two relations: equidistance relation $D$ and betweenness relation $B$. From this point of view, the class of all the metric spaces over ordered groups seems to be too large on the one hand, and too small on the other; too large - as regards geometric properties, and too small - as regards algebraic ones. For this reason, the objects under consideration are some special metric spaces over rather general algebraic structures.

To the author's best knowledge, this is the first paper on this subject. Since many questions remain open, the author believes this is not the last one.

To avoid any confusion, we give definitions of all the algebraic notions used in the paper, even those which can be found in the literature.

Algebraic preliminaries. Let us consider a system $\mathscr{G}=\left(G, G_{0}, 0,+\right)$ with $G_{0} \subset G-\{0\}$. We shall use $\alpha, \beta, \gamma, \ldots$ to denote elements of $G_{0}$ and $x, \lambda, \mu, \ldots$ to denote arbitrary elements of $G$.

The system $\mathscr{G}$ is said to be a commutative semi-group generated by $G_{0}$ whenever

0 is a neutral element with respect to + ,

+ is associative and commutative, and

$$
\bigwedge_{\lambda \neq 0} \bigvee_{\alpha_{1}, \ldots, \alpha_{k}} \lambda=\alpha_{1}+\ldots+\alpha_{k} .
$$

$\mathscr{G}$ is a commutative semi-group with cancellation if additionally

$$
\lambda+\mu=\lambda^{\prime}+\mu \Rightarrow \lambda=\lambda^{\prime} \quad \text { for every } \lambda, \lambda^{\prime}, \mu \text {. }
$$

* This paper was prepared at the Semingr-or Foundations of Geometry conducted by Professor W. Szmielew in 1973-74.

2 - Fundamenta Mathematicae XCVI 
Let $N$ be the set of natural numbers and let $\bar{N}=N \cup\{0\}$. Put

$$
0 \cdot \lambda \underset{\mathrm{df}}{=0} \quad \text { and } n \cdot \lambda \underset{\mathrm{df}}{=}(n-1) \cdot \lambda+\lambda \quad \text { for } \quad n \in N, \lambda \in G .
$$

$\mathscr{G}$ is freely generated by $G_{0}$ whenever

$$
\bigwedge_{\neq\left(\alpha_{1}, \ldots, \alpha_{k}\right)} \bigwedge_{\substack{m_{1}, \ldots, m_{k} \in \bar{N} \\ n_{1}, \ldots, n_{k} \in \bar{N}}}\left(m_{1} \alpha_{1}+\ldots+m_{k} \alpha_{k}=n_{1} \alpha_{1}+\ldots+n_{k} \alpha_{k} \Rightarrow m_{i}=n_{i}, i=1, \ldots, k\right) .
$$

EXAMPLE 1 . The semi-group $\mathscr{N}=(N,\{1\}, 0,+)$ is freely generated by $\{1\}$.

EXAMple 2. Given an arbitrary set $X_{0}$, let us consider the following set of functions:

$$
C \underset{\mathrm{df}}{=}\left\{\lambda \in \bar{N}^{X_{0}}: \lambda(\alpha)=0 \text { for almost all } \alpha \in X_{0}\right\} .
$$

Let

$$
(\lambda+\mu)(\alpha) \underset{\text { df }}{=} \lambda(\alpha)+\mu(\alpha) \quad \text { for every } \alpha \in X_{0} ;
$$

of course, setting

$$
\lambda=0 \underset{\mathrm{df}}{\Leftrightarrow} \lambda(\alpha)=0 \text { for every } \alpha \in X_{0}
$$

we get a neutral element of + . Take the subset $C_{0}$ of $C$ consisting of the non-zero characteristic functions for all the elements of $X_{0}$. The system

$$
\mathscr{C}\left(X_{0}\right) \underset{\mathrm{df}}{=}\left(C, C_{0}, 0,+\right)
$$

is a commutative semi-group freely generated by $C_{0}$. This semi-group will be referred to as the semi-group of chains in $X_{0}$ (over the semi-group $\mathcal{N}$ ) ${ }^{1}$ ).

According to the traditional notation we shall not distinguish between an element $\alpha$ of $X_{0}$ and its characteristic function, and thus we shall write $\lambda=\sum_{i=1}^{k} m_{i} \alpha_{i}$ whenever $\lambda\left(\alpha_{i}\right)=m_{i}$ for $i=1, \ldots, k$ and $\lambda(\alpha)=0$ otherwise.

A commutative semi-group is said to be free whenever it is freely generated by some $G_{0}$; it is said to be acyclic whenever

Let us notice that

$$
n \cdot \lambda=0 \Rightarrow n=0 \vee \lambda=0 .
$$

Proposition 1. Every free commutative semi-group is acyclic.

A system $(G, \geqslant)$ is a partially pseudo-ordered set provided $\geqslant$ is reflexive and weakly antysymmetric, i.e.

$$
\lambda \geqslant \lambda \text { and } \lambda \geqslant \lambda^{\prime} \wedge \lambda^{\prime} \geqslant \lambda \Rightarrow \lambda=\lambda^{\prime} \text { for every } \lambda, \lambda^{\prime} .
$$

( $\left.{ }^{1}\right)$ It is a natural modification of the well known notion of a group of chains over the group of integers.
A system $\mathscr{G}^{\geqslant}=(\mathscr{G}, \geqslant)$ is a partially pseudo-ordered semi-group whenever

I. $\mathscr{G}$ is a commutative semi-group,

II. $(G, \geqslant)$ is a partially pseudo-ordered set,

III. $1 . \lambda \geqslant 0$ for every $\lambda \in G$,

2. $\lambda \geqslant \lambda^{\prime} \Rightarrow \lambda+x \geqslant \lambda^{\prime}+x$, i.e. + is monotone with respect to $\geqslant$.

By III.1, 2 together with the weak antysymmetry of $\geqslant$, it follows that

$$
\lambda+\mu=0 \Rightarrow \lambda=0 \wedge \mu=0 \quad \text { for every } \lambda, \mu \text {; }
$$

thus

Propostrion 2. Every partially pseudo-ordered semi-group is acyclic.

Equidistance and betweenness relations in arbitrary metric spaces. Let us consider the class

$\mathbf{G}_{0}=\left\{\mathscr{G}^{\geqslant}=(\mathscr{G}, \geqslant): \mathscr{G} \geqslant\right.$ is a partially pseudo-ordered semi-group with cancellation $\}$ and a system

$$
\mathscr{X}=\left(X, \mathscr{G}^{\geqslant}, \varrho\right)
$$

consisting of a set $X$, a semi-group $\mathscr{G} \geqslant \in \mathbf{G}_{0}$ and a function $\varrho: X \times X \rightarrow G$ satisfying the well known metric axioms $\left({ }^{2}\right)$ :

M.1. $\varrho(a b)=0 \Leftrightarrow a=b$,

M.2. $\varrho(a b)=\varrho(b a)$,

M.3. $\varrho(a b)+\varrho(b c) \geqslant \varrho(a c)$.

The system $\mathscr{X}$ will be referred to as a metric space over $\mathscr{G} \geqslant$.

Let us define the following two relations in $\mathscr{X}$ :

$$
\begin{aligned}
& D_{\mathscr{X}}(a b c d) \underset{\mathrm{df}}{\Leftrightarrow} \varrho(a b)=\varrho(c d), \\
& B_{\mathscr{X}}(a b c) \underset{\mathrm{df}}{\leftrightarrow} \varrho(a b)+\varrho(b c)=\varrho(a c) .
\end{aligned}
$$

These two relations will be referred to as metric equidistance and metric betweenness relation.

Let $\mathbf{G} \subset \mathbf{G}_{0}$. Given an arbitrary class $\mathbf{M}$ of metric spaces over $\mathbf{G}$ (i.e. over semigroups from $\mathbf{G}$ ), one can consider the following three classes of relational structures:

$$
\begin{gathered}
\mathbf{D}_{\mathbf{M}}=\left\{\left(X, D_{\mathscr{x}}\right): \mathscr{X} \in \mathbf{M}\right\}, \quad \mathbf{B}_{\mathbf{M}}=\left\{\left(X, B_{\mathscr{x}}\right): \mathscr{X} \in \mathbf{M}\right\}, \\
\mathbf{D B}_{\mathbf{M}}=\left\{\left(X, D_{\mathscr{X}}, B_{\mathscr{x}}\right): \mathscr{X} \in \mathbf{M}\right\} .
\end{gathered}
$$

The problem arises, for which $\mathbf{M}$ the classes $\mathbf{D}_{\mathbf{M}}, \mathbf{B}_{\mathbf{M}}$ and $\mathbf{D B}_{\mathbf{M}}$ are elementary classes. We give a solution for $\mathbf{D B}_{\mathbf{M}_{0}}$ and $\mathbf{D}_{\mathbf{M}_{1}}$, the classes $\mathbf{M}_{0}$ and $\mathbf{M}_{1}$ being defined as follows.

$\left({ }^{2}\right)$ Throughout the paper we omit the universal quantifiers which should be placed in front of a formula to bound all the free variables occurring in it. 
Consider two additional metric axioms M.4 $4_{n}(n \in N)$ and M.5 (the first one is an elementary schema $\left.\left({ }^{3}\right)\right)$ :

$$
\begin{aligned}
& \text { M.4. } 4_{n} . \bigwedge_{\substack{a_{1} \ldots a_{n} \\
b_{1} \ldots b_{n}}} \bigvee_{\substack{q_{0} \ldots q_{n} \\
f:\{1, \ldots, n\} \rightarrow\{1, \ldots, n\}}} \bigvee_{\substack{1-1 \\
\text { s. }}}\left(\varrho\left(q_{0} q_{i-1}\right)+\varrho\left(q_{i-1} q_{i}\right)=\varrho\left(q_{0} q_{i}\right) \wedge\right. \\
& \left.\wedge \varrho\left(q_{i-1} q_{i}\right)=\varrho\left(a_{f^{\prime}(l)} b_{f(i)}\right)\right) . \\
& \Rightarrow \bigvee_{b^{\prime}}\left(\varrho(a b)=\varrho\left(a^{\prime} b^{\prime}\right) \wedge \varrho(b c)=\varrho\left(b^{\prime} c^{\prime}\right)\right)
\end{aligned}
$$

(The first sentenco describes a kind of rectifiability, the second one - a kind of homogenity.)

$\mathscr{X} \in \mathbf{M}_{0}$ whenever $\mathscr{G} \geqslant \in G_{0}$ and all the axioms M.1-M.5 are satisfied. Such a space $\mathscr{X}$ will be referred to as a regular metric space.

Let $\mathbf{G}_{1}=\left\{\mathscr{G} \geqslant \in \mathbf{G}_{0}: \mathscr{G} \geqslant\right.$ is a partially ordered free commutative semi-group $\}$.

$\mathscr{X} \in \mathbf{M}_{1}$ whenever $\mathscr{G} \geqslant \in \mathbf{G}_{1}$ and the axioms M.1-M.3 are satisfied.

Regular $D B$-structures. A $D B$-structure is understood as a model $(X, D, B)$ of the following axiom system (A.1-A.14 $)\left({ }^{4}\right)$ :

A.1. $\quad D(a b a b)$,

A.2. $D(a b c d) \wedge D\left(a^{\prime} b^{\prime} c d\right) \Rightarrow D\left(a b a^{\prime} b^{\prime}\right)$,

A.3. $\quad D(a a b b)$,

A.4. $D(a b c c) \Rightarrow a=b$,

A.5. $D(a b b a)$,

A.6. $B(a a b)$,

A.7. $B(a b c) \Rightarrow B(c b a)$,

A.8. $\quad B(a b c) \wedge B(a c b) \Rightarrow b=c$,

A.9. $B(a b d) \wedge B(b c d) \Rightarrow B(a b c) \wedge B(a c d)$,

A.10. $B(a b d) \wedge B(b c d) \wedge D(a d b c) \Rightarrow a=b \wedge c=d$,

A.11. $\quad D\left(a b a^{\prime} b^{\prime}\right) \wedge D\left(b c b^{\prime} c^{\prime}\right) \wedge D\left(a c a^{\prime} c^{\prime}\right) \wedge B(a b c) \Rightarrow B\left(a^{\prime} b^{\prime} c^{\prime}\right)$,

A.12. $D\left(a b a^{\prime} b^{\prime}\right) \wedge D\left(a c a^{\prime} c^{\prime}\right) \wedge B(a b c) \wedge B\left(a^{\prime} b^{\prime} c^{\prime}\right) \Rightarrow D\left(b c b^{\prime} c^{\prime}\right)$,

$\mathrm{A} \cdot 13_{n} . \bigwedge_{i=1}^{n}\left[B\left(p_{0} p_{i-1} p_{i}\right) \wedge B\left(q_{0} q_{i-1} q_{i}\right)\right] \wedge \bigvee_{f:\{1, \ldots, n\} \rightarrow\{1, \ldots, n\}} D\left(p_{i-1} p_{i} q_{f(i)-1} q_{f(i)}\right)$

$$
\Rightarrow D\left(p_{0} p_{n} q_{0} q_{n}\right)\left(^{5}\right)
$$

(3) The symbol $\underset{f:\{1, \ldots, n\} \rightarrow\{1, \ldots, n)}{\mathrm{V}}$ denotes an alternative of $n$ ! formulae.

(4) See footnote ${ }^{(3)}$.

(5) We omit “ $\bigwedge_{i=1}^{n} "$ if it does not cause any misunderstanding.

A. $14_{n} . \quad \bigwedge_{i=1}^{m} \bigwedge_{j=1}^{n}\left[B\left(p_{0} p_{i-1} p_{i}\right) \wedge B\left(q_{0} q_{j-1} q_{j}\right)\right] \wedge\left(p_{0}, p_{m}, q_{0}, q_{n}\right)=\left(p_{0}^{\prime}, p_{n}^{\prime}, q_{0}^{\prime}, q_{m}^{\prime}\right) \wedge$

$$
\wedge \underset{\substack{f: 1 \\ f:\{1, \ldots, m\} \rightarrow\{1, \ldots, m\} \\ g:\{1, \ldots, n\} \rightarrow\{1, \ldots, n\}}}{\bigvee}\left[D\left(p_{i-1} p_{i} q_{f(i)-1}^{\prime} q_{f(i)}^{\prime}\right) \wedge D\left(q_{j-1} q_{j} p_{g(j)-1}^{\prime} p_{g(j)}^{\prime}\right)\right]
$$

A $D B$-structure is said to be regular provided it satisfies additionally the following two axioms A.15n and A.16:

A.15n. $\bigwedge_{\substack{a_{1}, \ldots, a_{n} \\ b_{1}, \ldots, b_{n}}} \bigvee_{q_{0}, \ldots, q_{n}} \bigvee_{f:\{1, \ldots, n\} \rightarrow\{1, \ldots, n\}}\left[B\left(q_{0} q_{i-1} q_{i}\right) \wedge D\left(q_{i-1} q_{i} a_{f(i)} b_{f(i)}\right)\right]$,

A.16. $\quad B(a b c) \wedge D\left(a c a^{\prime} c^{\prime}\right) \Rightarrow \bigvee_{b^{\prime}}\left[D\left(a b a^{\prime} b^{\prime}\right) \wedge D\left(b c b^{\prime} c^{\prime}\right)\right]$

As direct consequences of the above axioms one obtains

0.1. $B(a b a) \Rightarrow a=b \quad$ (by A.6, A.8),

$0.2_{n} . \quad B\left(a b p_{n+1}\right) \wedge \bigwedge_{i=1}^{n} B\left(b p_{i} p_{i+1}\right) \Rightarrow \bigwedge_{i=1}^{n} B\left(a p_{i} p_{i+1}\right) \quad$ (by A.7, A.9)

$0.3_{n} . \quad \bigwedge_{i=1}^{n} B\left(p_{0} p_{i-1} p_{i}\right) \Rightarrow \bigwedge_{0<i<j \leqslant n} B\left(p_{0} p_{i} p_{j}\right) \quad($ by A.7, A.9),

$0.4_{n} . \quad \bigwedge_{i=1}^{n} B\left(p_{0} p_{i-1} p_{i}\right) \wedge D\left(p_{0} p_{n} q_{0} q_{n}\right)$

$\Rightarrow \bigvee_{q_{1}, \ldots, q_{n-1}}\left[B\left(q_{0} q_{i-1} q_{i}\right) \wedge D\left(p_{0} p_{i-1} q_{0} q_{i-1}\right) \wedge D\left(p_{i-1} p_{i} q_{i-1} q_{i}\right)\right]$

(by A.11, A.16).

Theory of equidistance and betweenness relations in regular metric spaces. We are going to prove the following

REPRESENTATION THEOREM I. The class $\mathbf{D B}_{\mathbf{M}_{0}}$ coincides with the class of models of (A.1-A.16), i.e.

$$
\mathbf{D B}_{\mathbf{M}_{0}}=\mathfrak{M}(\mathrm{A} .1-\mathrm{A} .16)
$$

This theorem is a corollary of Theorems I.1 and I.2 below.

THEOREM I.1. Every structure $\left(X, D_{\mathscr{X}}, B_{\mathscr{X}}\right)$, with $\mathscr{X} \in \mathbf{M}_{0}$, is a model of (A.1-A.16).

Proof. Let $\mathscr{X}=\left(X, \mathscr{G}^{\geqslant}, \varrho\right) \in \mathbf{M}_{0}$; then, by Proposition $2, \mathscr{G} \geqslant$ is acyclic. The structure $\left(X, D_{\mathscr{x}}, B_{\mathscr{X}}\right)$ satisfies A.1-A.16; indeed, A.1, A.2, A.11 and A.13 follow by the definitions of $D_{x}$ and $B_{\mathscr{X}} ; \mathrm{A} .3-\mathrm{A} .5$ by M.1 and M.2;A.6 - by $0+\lambda=\lambda$; M.2 and commutativity of + imply A.7; the condition M.2 together with acyclicity and cancellation imply A.8; in turn A.9 follows by M.3 together with cancellation, monotony of + and weak antysymmetry of $\geqslant ;$ A.10 - by M.1, acyclicity and cancellation; A.12 - by cancellation; A.14 $n_{n}$ by M.3, commutativity of + and weak antysymmetry of $\geqslant ; \mathrm{A} .15_{n}$ - by M. $4_{n}$, and finally A.16 - by M.5. 
Theorem I.1 enables us to define the following function

$$
\begin{gathered}
\Phi_{0}: \mathbf{M}_{0} \rightarrow \mathfrak{M}(\text { A.1-A.16), } \\
\Phi_{0}(\mathscr{X}) \underset{\mathrm{df}}{=}\left(X, D_{\mathscr{X}}, B_{\mathscr{X}}\right) \quad \text { for every } \mathscr{X} \in \mathbf{M}_{0} .
\end{gathered}
$$

THeOREM I.2. For every regular. DB-structure $(X, D, B)$ there is a regular metric space $\mathscr{X}$ such that $D_{\mathscr{X}}=D$ and $B_{\mathscr{X}}=B$.

In other words, there is a function

$$
\Psi_{0}: \mathfrak{M}(\mathrm{A} .1-\mathrm{A} .16) \rightarrow \mathrm{M}_{0}
$$

such that $\Phi_{0} \Psi_{0}(X, D, B)=(X, D, B)$ for every $(X, D, B) \in \mathfrak{M}$ (A.1-A.16).

The construction of the function $\Psi_{0}$ will be referred to as a metrization of regular DB-structures.

Metrization of regular $D B$-structures. Let us consider an arbitrary regular $D B$-structure, i.e. any model $(X, D, B)$ of the axiom system (A.1-A.16). We are going to define a partially pseudo-ordered semi-group with cancellation, $\mathscr{G}_{(X, D, B)}^{\geqslant}=\left(\mathscr{G}_{(X, D, B)}, \geqslant\right)$, and a function $\varrho: X \times X \rightarrow G_{(X, D, B)}$ such that the system $\mathscr{X}=\left(X, \mathscr{G}_{(X, D, B)}^{\geqslant}, \varrho\right)$ is a regular metric space, and the relations $D_{\mathscr{X}}$ and $B_{\mathscr{X}}$ coincide with $D$ and $B$.

1. The quaternary relation $D$ in $X$ induces the following binary relation $\equiv \operatorname{in} X^{2}$; 1.0 .

$$
a b \equiv c d \underset{\mathrm{df}}{\Leftrightarrow} D(a b c d) \text {. }
$$

By A.1-A.5 we get

1.1. $\equiv$ is an equivalence relation,

1.2. $a a \equiv b b$,

1.3. $a b \equiv c c \Rightarrow a=b$,

1.4. $a b \equiv b a$.

Let $\left.X_{0} \underset{\mathrm{df}}{=} X^{2}\right|_{\equiv}$. Consider the semi-group of chains $C\left(X_{0}\right)$ (see Example 2) and let $\doteq$ be the smallest congruence in $C\left(X_{0}\right)$ which contains $\{([a a], 0)\}$. Let

$$
\mathscr{G}_{(X, D)} \underset{\mathrm{df}}{=} C\left(X_{0}\right) \mid \doteq \text {. }
$$

\section{Notice that}

1.5. $\mathscr{G}_{(X, D)}$ is a commutative semi-group freely generated by $X_{0} \mid \doteq$.

2. We are going now to define (by means of $B$ ) a binary relation $\sim_{B}$ in $G_{(X, D)}$, satisfying the following five conditions:

(i) $\sim_{B}$ is a congruence with respect to + , (ii) $\lambda \underset{B}{\sim} 0 \Leftrightarrow \lambda=0$,

(iii) $\quad \underset{B}{\sim} \beta \Leftrightarrow \alpha=\beta$,

(iv) $[a b]+[b c] \underset{B}{\sim}[a c] \Leftrightarrow B(a b c)$,

(v) $\lambda+\beta \underset{B}{\sim} \alpha+\beta \Rightarrow \lambda \underset{B}{\sim} \alpha$.

Let us consider the following function $S: G_{(X, D)} \rightarrow 2^{G_{(X, D)}}$

$S(\alpha) \underset{\mathrm{df}}{=}\left\{\left[p_{0} p_{1}\right]+\ldots+\left[p_{n-1} p_{n}\right]: \alpha=\left[p_{0} p_{n}\right] \wedge B\left(p_{0} p_{i-1} p_{i}\right), i=1, \ldots, n, n \in N\right\}$,

2.0. $S\left(\sum_{i=1}^{k} m_{i} \alpha_{i}\right) \underset{\mathrm{df}}{=}\left\{\sum_{i=1}^{k} m_{i} \lambda_{i}: \lambda_{i} \in S\left(\alpha_{i}\right), i=1, \ldots, k\right\}$.

The relation $\underset{B}{\sim}$ is defined by the formula

$$
\lambda \underset{B}{\sim} \lambda^{\prime} \underset{\mathrm{df}}{\Leftrightarrow} \bigvee_{x} \lambda, \lambda^{\prime} \in S(x) .
$$

In the sequel, we write simply $\sim$ whenever there is no danger of a confusion. The proof of (i)-(v) is based on the following statements concerning $S$.

2.1. $S(0)=\{0\}$,

2.2. $0 \in S(\lambda) \Rightarrow \lambda=0$ (by 1.5),

2.3. $\lambda \in S(\lambda)$ (by A.6),

2.4. $\lambda \in S(\alpha) \Leftrightarrow \bigvee_{n \in N \neq\left(p_{0} \ldots p_{n}\right)}\left(\lambda=\left[p_{0} p_{1}\right]+\ldots+\left[p_{n-1} p_{n}\right] \wedge \alpha=\left[p_{0} p_{n}\right] \wedge B\left(p_{0} p_{i-1} p_{i}\right)\right.$

2.5. $x \in S\left(\lambda_{1}+\lambda_{2}\right) \Rightarrow \bigvee_{x_{1}, x_{2}}\left(x=x_{1}+x_{2} \wedge x_{i} \in S\left(\lambda_{i}\right)\right)$

2.6. $S(\alpha) \cap S(\beta) \neq \varnothing \Rightarrow \alpha=\beta \quad$ (by $2.4,1.5$ and $\mathrm{A} .13_{n}$ ),

2.7. $\alpha \in S(x) \Rightarrow x=\alpha \quad$ (by $2.2,2.3,2.5,2.6$ ),

2.8. $x \in S(\lambda) \wedge \lambda \in S(\mu) \Rightarrow x \in S(\mu) \quad$ (by $2.4,2.5,1.5,0.4_{n}$ and $0.2_{n}$ )

Proof. Let $\lambda=\sum_{i=1}^{l} \alpha_{i}$ and $\mu=\sum_{j=1}^{m} \beta_{j}$. Induction on $m$.

$1^{0}$ Let $m=1$, then

$$
\lambda \in S(\mu) \Rightarrow \sum_{i=1}^{l} \alpha_{i} \in S\left(\beta_{1}\right)
$$

by 2.5 ,

$$
x \in S(\lambda) \Rightarrow \bigvee_{x_{1}, \ldots, x_{1}}\left(x=\sum_{i=1}^{l} x_{i} \wedge x_{i} \in S\left(\alpha_{i}\right)\right)
$$

By 2.4,

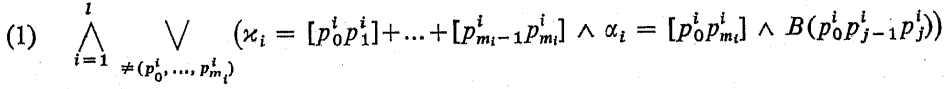


and

(2) $\underset{\neq\left(q 0, \ldots, q^{n}\right)}{\bigvee}\left(\sum_{i=1}^{l} \alpha_{i}=\left[q^{0} q^{1}\right]+\ldots+\left[q^{n-1} q^{n}\right] \wedge \beta_{1}=\left[q^{0} q^{n}\right] \wedge B\left(q^{0} q^{j-1} q^{j}\right)\right)$.

Thus, by 1.5 , one gets

(3) $l=n$ and there is a bijection $f:\{1, \ldots, n\} \rightarrow\{1, \ldots, n\}$ such that $\left[p_{0}^{i} p_{m_{i}}^{i}\right]=\left[q^{f(i)-1} q^{f(i)}\right]$ for $i=1, \ldots, n$.

By (1), (3) and $0.4_{m_{k}}(k=1, \ldots, n)$, there exist $q_{0}^{k}, \ldots, q_{m_{k}}^{k}$ such that

(4) $B\left(q_{0}^{k} q_{j-1}^{k} q_{j}^{k}\right), \quad q_{j-1}^{k} q_{j}^{k} \equiv p_{j-1}^{i} p_{j}^{i}$ for $j=1, \ldots, m_{i}=m_{k}, \quad k=f(i)$, and $q_{0}^{k}=q^{k-1}, \quad q_{m_{k}}^{k}=q^{k}$ for $k=1, \ldots, n$.

By (2)-(4), applying $0.2_{n}$, one gets

$$
B\left(q^{0} q_{j-1}^{k} q_{j}^{k}\right) \text { for } j=1, \ldots, m_{k}, k=1, \ldots, n .
$$

By (1)-(5), $x \in S\left(\beta_{1}\right)$, i.e. $x \in S(\mu)$ for $m=1$.

$2^{0}$ Assume that the assertion holds for $m \leqslant m_{0}-1$ and let $m=m_{0}$, i.e. $\mu=\sum_{i=1}^{m_{0}} \beta_{i}$. Let $\mu^{\prime}=\sum_{i=1}^{m_{0}-1} \beta_{i}$; then $\mu=\mu^{\prime}+\beta_{m_{0}}$. Since $\lambda \in S(\mu)$, by 2.5 it follows that

$$
\underset{\lambda^{\prime}, \lambda^{\prime \prime}}{\bigvee} \lambda=\lambda^{\prime}+\lambda^{\prime \prime} \wedge \lambda^{\prime} \in S\left(\mu^{\prime}\right) \wedge \lambda^{\prime \prime} \in S\left(\beta_{m_{0}}\right)
$$

in turn, since $x \in S\left(\lambda^{\prime}+\lambda^{\prime \prime}\right)$, hence

$$
\bigvee_{x^{\prime}, x^{\prime \prime}} x=x^{\prime}+x^{\prime \prime} \wedge x^{\prime} \in S\left(\lambda^{\prime}\right) \wedge x^{\prime \prime} \in S\left(\lambda^{\prime \prime}\right) \text {. }
$$

By the inductive assumption, (6) and (7) imply $x^{\prime} \in S\left(\mu^{\prime}\right)$ and $x^{\prime \prime} \in S\left(\beta_{m_{0}}\right)$, thus $x \in S(\mu)$.

As a direct consequence of A.15, one obtains

2.9. $\bigwedge_{x \neq 0} \bigvee_{\alpha} x \in S(\alpha)$.

The statements 2.1-2.9 imply the following assertions concerning the relation $\sim$.

2.10. $\lambda \sim \lambda^{\prime} \Leftrightarrow \bigvee_{\alpha} \lambda, \lambda^{\prime} \in S(\alpha) \quad$ (by $2.8,2.9$ ),

2.11. $\lambda \sim \beta \Rightarrow \lambda \in S(\beta)$ (by $2.9,2.3$ and 2.6 ).

By 2.10 and 1.5 together with A.12, it follows easily that

2.12. $\alpha^{\prime}+\beta \sim \alpha+\beta \Rightarrow \alpha^{\prime}=\alpha$.

Now, the condition (i) follows by $2.0,2.3,2.5,2.6,2.10$; condition (ii) - by 2.1 , 2.2 ; the condition (iii) - by 2.7 .
Proof of (iv): (by 2.11, 2.4, A.5-A.7, A.11, 2.0, 2.3). Let $[a b]+[b c] \sim[a c]$. If either $a=b$ or $b=c$, then by A.6 and A.7 one gets $B(a b c)$. If $a=c$, then, by (ii), $a=b$ and thus $B(a b c)$. If $\neq(a b c)$, then, by $2.11,[a b]+[b c] \in S([a c])$. By 2.4, there exist $\neq\left(a^{\prime} b^{\prime} c^{\prime}\right)$ such that $[a b]+[b c]=\left[a^{\prime} b^{\prime}\right]+\left[b^{\prime} c^{\prime}\right],[a c]=\left[a^{\prime} c^{\prime}\right]$ and $B\left(a^{\prime} b^{\prime} c^{\prime}\right)$. There are two possibilities: either $a b \equiv a^{\prime} b^{\prime}$ and $b c \equiv b^{\prime} c^{\prime}$ or $a b \equiv b^{\prime} c^{\prime}$ and $b c \equiv a^{\prime} b^{\prime}$. In the first case A.11 implies $B(a b c)$, in the second one, A.5 and A.11 imply $B(a b c)$. The converse implication $B(a b c) \Rightarrow[a b]+[b c] \sim[a c]$ follows directly by 2.0 and 2.3 .

Pro of of (v): (by 2.8, 2.10, 2.3, 2.6, and 2.12). Let $\lambda+\beta \sim \alpha+\beta$. By 2.10, there is a $\gamma$ such that $\lambda+\beta \in S(\gamma)$ and $\alpha+\beta \in S(\gamma)$. Thus, there are $p_{0}, \ldots, p_{n}$ such that $B\left(p_{0} p_{i-1} p_{i}\right)$ for $i=1, \ldots, n, \lambda+\beta=\left[p_{0} p_{1}\right]+\ldots+\left[p_{n-1} p_{n}\right]$ and $\gamma=\left[p_{0} p_{n}\right]$. Then, by 1.5 , there is a $j \in\{1, \ldots, n\}$ such that $\beta=\left[p_{j-1} p_{j}\right]$. Let

Obviously

$$
\varkappa=\left[p_{0} p_{j-1}\right]+\left[p_{j} p_{n}\right] \text {. }
$$

$$
\lambda \in S(x)
$$

By 2.5 , there exist $\alpha^{\prime}$ and $\gamma^{\prime}$ such that

$$
x \in S\left(\alpha^{\prime}\right)
$$

and

$$
\alpha^{\prime}+\beta \in S\left(\gamma^{\prime}\right)
$$

By (1), (2) and 2.3 , one gets

$$
\text { (4) } \lambda+\beta \in S(x+\beta) \text { and } x+\beta \in S\left(\alpha^{\prime}+\beta\right) \text {. }
$$

By 2.8, the condition (4) implies

$$
\lambda+\beta \in S\left(\alpha^{\prime}+\beta\right),
$$

and (5) together with (3) imply $\lambda+\beta \in S\left(\gamma^{\prime}\right)$. By $2.6, \lambda+\beta \in S(\gamma) \cap S\left(\gamma^{\prime}\right)$ implies $\gamma^{\prime}=\gamma$. Thus, by (3), $\alpha^{\prime}+\beta \in S(\gamma)$ and $\alpha+\beta \in S(\gamma)$, i.e. $\alpha^{\prime}+\beta \sim \alpha+\beta$. Then by 2.12 , $\alpha^{\prime}=\alpha$ and thus, by (1), (2) and 2.8, $\lambda \in S(\alpha)$. Therefore $\lambda \sim \alpha$.

3. We are going now to define a binary relation $\geqslant$ in $G_{(X, D)}$, satisfying the following six conditions:

(j) $\lambda \geqslant 0$ for every $\lambda$,

(ji) $\geqslant$ is reflexive,

(jjj) $\widetilde{\sim}_{B}$ is a congruence with respect to $\geqslant_{B}$,

(jw) + is monotone with respect to $\geqslant$,

(w) $\lambda \geqslant_{B} x \wedge x \geqslant \lambda \Rightarrow x_{B} \lambda$,

(wj) $[a b]+[b c] \geqslant[a c]$. 
Let us consider the following function $T: G_{(X, D)} \rightarrow 2^{G_{(X, D)}}$

$$
T(0) \underset{\mathrm{df}}{=} G_{(X, D)},
$$

3.0. $T(\alpha) \underset{\mathrm{df}}{=}\left\{\left[p_{0} p_{1}\right]+\ldots+\left[p_{n-1} p_{n}\right]: \alpha=\left[p_{0} p_{n}\right], n \in N\right\}$,

$$
T\left(\sum_{i=1}^{k} m_{i} \alpha_{i}\right) \underset{\mathrm{df}}{=}\left\{\sum_{i=1}^{k} m_{i} \lambda_{i}: \lambda_{i} \in T\left(\alpha_{i}\right), i=1, \ldots, k\right\} \text { for } m_{i} \in N, i=1, \ldots, k \text {. }
$$

The relation $\geqslant_{B}$ is defined by means of the auxiliary relation $\geqslant$ :

$$
\begin{aligned}
& \lambda \geqslant x \underset{\mathrm{df}}{\Leftrightarrow} \bigvee_{\mu}(\lambda \in T(\mu) \wedge x \in S(\mu)), \\
& \lambda \geqslant_{B} x \underset{\text { df }}{\bigvee_{\lambda^{\prime}}}\left(\lambda^{\prime} \sim \lambda \wedge \lambda^{\prime} \geqslant x\right) \text {. }
\end{aligned}
$$

Obviously

3.1. $S(\lambda) \subset T(\lambda)$,

3.2. $\lambda \in T(\lambda)$,

and

3.3. $x \in T(\lambda) \wedge \lambda \in T(\mu) \Rightarrow x \in T(\mu)$.

As a consequence of A.14 and $0.4_{n}$ one gets

3.4. $S(\alpha) \cap T(\beta) \neq \varnothing \neq T(\alpha) \cap S(\beta) \Rightarrow \alpha=\beta$.

By $2.9,2.8,3.1$ and 3.3 it follows that

3.5. $\lambda \geqslant x \Leftrightarrow \bigvee_{\alpha} \lambda \in T(\alpha) \wedge x \in S(\alpha)$.

The condition (j) follows by 3.0 and 2.1 together with the reflexivity of $\underset{\boldsymbol{B}}{\sim}$; the condition (jj) - by 3.2 and 2.3 ; the condition (jj) - by 2.10, 3.5 and 2.6; the additivity of $S$ and $T$ implies (jw); the statements 2.6, 2.10 and 3.4 imply (w); the condition (wj) follows immediately by 2.0 .

4. The condition (i) enables us to define the semi-group $\mathscr{G}_{(X, D, B)}$ as the quotient algebra:

$$
\mathscr{G}_{(X, D, B)}=\left.\mathscr{G}_{(X, D)}\right|_{B} ^{\sim} .
$$

By (ii), the class [0] is the neutral element of $\mathscr{G}_{(X, D, B)}$. The condition (v) together with 2.9 imply the cancellation law. Thus

4.1. $\mathscr{G}_{(X, D, B)}$ is a commutative semi-group with cancellation.

The condition (jjj) enables us to define the relation $\geqslant$ in $\mathscr{G}_{(X, D, B)}$ by the formula

$$
[\lambda]_{\sim} \geqslant[x]_{\sim} \underset{\mathrm{df}}{\Leftrightarrow} \lambda \geqslant x .
$$

Let

$$
\mathscr{G}_{(X, D, B)}^{\geqslant}=\left(\mathscr{G}_{(X, D, B)}, \geqslant\right) .
$$

By (j), (jj) and (w), $\geqslant$ is a partial pseudo-order in $G_{(X, D, B)}$. By (jw), + is monotone with respect to $\geqslant$. Thus

4.2. $\mathscr{G}_{(X, D, B)}^{\geq}$is a partially pseudo-ordered semi-group with cancellation.

Let us define the function $\varrho: X \times X \rightarrow G_{(X, D, B)}$ by the formula

$$
Q(a b) \underset{\text { df }}{=}[[a b]]_{\sim}
$$

and let $\mathscr{X} \underset{\text { df }}{=}\left(X, \mathscr{G}_{(X, D, B)}^{\geqslant}, \varrho\right)$. It is easy to show (applying (ii), 1.4 and (wj)) that $\mathscr{X}$ satisfies M.1-M.3; thus

4.3. $\mathscr{X}$ is a metric space over $\mathscr{G}_{(X, D, B)}^{\geqslant}$.

Let us consider now the metric equidistance relation $D_{\mathscr{X}}$ and metric betweenness relation $B_{\mathscr{X}}$. The conditions (iii) and (iv) imply

4.4. $\quad D_{\mathscr{X}}^{\top}=D$ and $B_{\mathscr{X}}=B$.

By 4.4, the axioms A.15 ${ }_{n}$ and A.16 are equivalent respectively to M. $4_{n}$ and M.5. Hence

4.5. The metric space $\mathscr{X}$ is regular.

Proof of Theorem I.2. Define the function $\Psi_{0}: \mathfrak{M}\left(\right.$ A.1-A.16) $\rightarrow \mathbf{M}_{0}$ by the formula

$$
\Psi_{0}(X, D, B) \underset{\mathrm{df}}{=}\left(X, \mathscr{G}_{(X, D, B)}^{\geqslant}, \varrho\right)=\mathscr{X} .
$$

This function $\Psi_{0}$ satisfies the required condition; indeed,

$$
\Phi_{0} \Psi_{0}(X, D, B)=\Phi_{0}\left(X, \mathscr{C}_{(X, D, B)}^{\geqslant}, \varrho\right)=\left(X, D_{\mathscr{X}}, B_{\mathscr{x}}\right),
$$

thus, by $4.4, \Phi_{0} \Psi_{0}(X, D, B)=(X, D, B)$.

4.6. Remark. Let us notice that, for the metrization of a $D B$-structure, not the statements 2.0-2.11 and 3.0-3.5 themselves, but only the conditions (i)-(v) and (j)-(wj) are essential. More precisely, given two relations $\underset{B}{\sim}$ and $\underset{B}{\geqslant}$ in $G_{(X, D)}$ satisfying the above conditions, one can define $\mathscr{G}_{(X, D, B)}^{\geqslant}$and $\varrho$ as it was done in $\S 4$, and then, setting $\mathscr{X}=\left(X, \mathscr{G}_{(X, D, B)}^{\geqslant}, \varrho\right)$, one can prove $\mathscr{X}$ to be a metric space over $\mathbf{G}_{0}$, with $D_{x}=D$ and $B_{x}=B$

4.7. Remark. The axioms A.7-A.16 are needed only in the proofs of $2.4-2.11$, $3.4,3.5$ and in the proof of the regularity of $\mathscr{X}$. Since (j), (jj) and (jw) follow by 2.0-2.3 and 3.0-3.2, these three conditions require no axioms on $D, B$ but A.1-A.6. 
Theory of metric equidistance relation. Consider now the class $M_{1}$ of all the metric spaces over partially ordered free semi-groups. Applying the previous results, we are going to prove

REPRESENTATION THEOREM. II. The class $D_{\mathrm{M}_{1}}$ coincides with the class of models of (A.1-A.5), i.e.

$$
D_{M_{1}}=\mathfrak{M}(\text { A.1-A.5) }
$$

Since every partially ordered semi-group with cancellation (thus moreover every free semi-group) can be extended to an ordered group, this theorem can be formulated as follows:

The theory of equidistance relation in metric spaces over ordered groups coincides with the theory based on (A.1-A.5).

We have first the following obvious

THEOREM II.1. Every structure $\left(X, D_{\mathscr{x}}\right)$, with $\mathscr{X} \in \mathrm{M}_{1}$, is a model of (A.1-A.5) $\left({ }^{6}\right)$. This theorem enables us to define the following function

$$
\begin{gathered}
\Phi_{1}: M_{1} \rightarrow \mathfrak{M}(\text { A.1-A.5), } \\
\Phi_{1}(\mathscr{X}) \underset{\mathrm{d} f}{=}\left(X, D_{\mathscr{X}}\right) \quad \text { for every } \mathscr{X} \in \mathbb{M}_{1} .
\end{gathered}
$$

Now, it remains to prove

THEOREM II.2. For every model $(X, D)$ of (A.1-A.5) there is a metric space $\mathscr{X} \in \mathbf{M}_{1}$ such that $D_{\mathscr{C}}=D$.

In other words, there is a function $\Psi_{1}: \mathfrak{M}\left(\right.$ A.1-A.5) $\rightarrow M_{1}$ such that

$$
\Phi_{1} \Psi_{1}(X, D)=(X, D) \quad \text { for every }(X, D) \in \mathfrak{M}(\mathrm{A} .1-\mathrm{A} .5)
$$

Proof. Let $(X, D)$ be a model of (A.1-A.5). We are going to define the required metric space $\mathscr{X}=\left(X, \mathscr{G}^{\geqslant}, \varrho\right)$. Take $\mathscr{G}_{(X, D)}$ as constructed in $\S 1$. By $1.5, \mathscr{G}_{(X, D)}$ is a free semi-group. Let us extend the structure $(X, D)$ to a $D B$-structure $\left(X, D, B_{0}\right)$ setting

$$
B_{0}(a b c) \underset{\mathrm{df}}{\Leftrightarrow} a=b \vee b=c .
$$

Evidently, $\left(X, D, B_{0}\right)$ satisfies A.1-A.6 (moreover, it satisfies all the axioms except A. $15_{n}$ ).

Look at the relations $\underset{B_{0}}{\sim}$ and $\underset{B_{0}}{\geqslant}$ in $G_{(X, D)}$. First of them is the identity $=$, thus it satisfies the conditions (i)-(v) $(\S 2)$ and (ijj) (§3). The second one is of the form.

$$
\lambda \geqslant x \Leftrightarrow \lambda \in T(x)
$$

${ }^{\left({ }^{6}\right)}$ Here $\mathbf{M}_{1}$ can be replaced by any class of metric spaces over arbitrary semi-groups, even thus it satisfies the condition (wj). Since $\mathscr{G}_{(X, D)}$ is free, (w) follows by 3.0. By Remark 4.7 , conditions (j), (jj) and (jw) are also satisfied; moreover 3.3 implies the transitivity of $\geqslant$.

By Remark 4.6, setting

(2) $\mathscr{G}=\left.\underset{\mathrm{df}}{=} \mathscr{G}_{(X, D)}\right|_{\widetilde{B}_{0}}, \quad[\lambda] \geqslant[x] \underset{\mathrm{df}}{\Leftrightarrow} \lambda_{B_{0}} \geqslant, \quad \mathscr{G} \geqslant \underset{\mathrm{df}}{=}(\mathscr{G}, \geqslant) \quad$ and $\quad \varrho(a b) \underset{\mathrm{df}}{=}[[a b]]_{\widetilde{B_{0}}}$, one obtains the required metric space $\mathscr{X}$ over $\mathscr{G} \geqslant$. By $(2), \mathscr{G}=\mathscr{G}_{(X, D)}$ and $\geqslant$ coincides with $\geqslant$, thus $\mathscr{G} \geqslant$ is a partially ordered free semi-group. This completes the proof.

Remarks and problems. Consider the class $\mathbf{M}_{2}$ of all the metric spaces over $\mathbf{G}_{0}$. Every structure $\left(X, D_{\mathscr{X}}, B_{\mathscr{X}}\right)$, with $\mathscr{X} \in \mathrm{M}_{2}$, is a model of (A.1-A.14n $)$, i.e.

$$
D B_{\mathrm{M}_{2}} \subset \mathfrak{M}\left(\mathrm{A} \cdot 1-\mathrm{A} \cdot 14_{n}\right) \text {. }
$$

However, the author does not know whether the converse inclusion holds. The question remains open if replaced $M_{2}$ by the smaller class $M_{3}$ of all the metric spaces over partially ordered semi-groups with cancellation. The additional axioms A.15 and A.16 make the construction of $\mathscr{G}_{(X, D, B)}^{\geqslant}$rather simply, since they imply the useful statements 2.8-2.11 and 3.4-3.5. On the other hand, these axioms restrict considerably the class of involved metric spaces.

Our axiom system is far no perfect. Especially the schema A.14 (connected with the weak antysymmetry of $\geqslant$ ) looks complicated. The author has not studied exactly the independence of this axiom system; however it is clear that A.13 ${ }_{1}$ is a tautology and A.13 $3_{3}$ depends on (A.2, A.5, A.7, A.13 $_{2}$. The axiom A.10 depends on the other axioms including A.15 ; nevertheless this is not omitted, since, in fact, we are interested in the axiom system (A.1-A.14n) as well as in the whole system (A.1-A.16).

INSTITUTE OF MATHEMATICS UNIVERSITY OF WARSAW

Accepté par la Rédaction le 26. 4. 1975 without any ordering relation. 